influence of rotation on the moving liquid and particles, the centripetal part of the tube should be turned towards the direction of rotation. If two tubes with suitable chosen values of $R / L$ are coupled in series so that the tube with a larger $r_{\min }$. is first passed by the particles, any desired fraction of particles can be removed from the suspension.

In work with large particles having a high density compared to that of the liquid, and a low liquid viscosity, a necessary condition is that the influence of gravity on the particles is eliminated. This can be realized by rotating the centrifuge in the vertical plane.

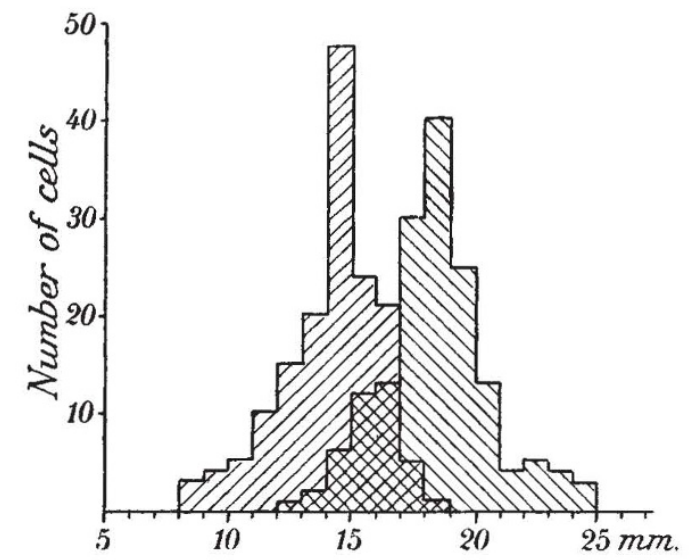

BAKER'S YEAST CELLS SUSPENDED IN TAP WATER AND CENTRIFUGED FOR 60 MINUTES. ABSCISSA, GREATEST DIAMETER OF THE ORLIS $(\times 2,350)$. SHADED AREAS SHOW NUMBERS OF LARGRR GRILS RETAINED IN THE SEPARATING TUBE AND IN A FOLLOWING TUBE RESPECTIVELY; THE OVERLAPPING WILI DIMTNISH ON PROLONGED OENTRIFUGATION

A trial centrifuge has been constructed on these principles and is at present being employed for the fractionation of yeast cells (see graph) and other biological material. A detailed description will shortly be published elsewhere.

Institute of Zoophysiology,

Per Eric lindahi

University of Uppsala.

Jan. 8.

\section{Electrokinetic Potential and Surface Structure of Barium Sulphate}

WHInE there are many investigations on the influence of electrolytes on the electrokinetic or $\zeta$-potential of solids, very few investigators have considered the relation between the $\zeta$-potential and the structure and composition of the solid itself. This is partly due to the fact that in many systems the potential-determining ions are foreign to the solid.

We are at present investigating the $\zeta$-potential of sparingly soluble ionic solids such as barium sulphate against their saturated solution by the streaming potential method. In this case, the potentialdetermining ions originate from the solid. Precipitated barium sulphate is unsuitable for investigation, because the validity of the electrokinetic equations is limited to systems in which the diameter of the 'pores' of a plug is greater than the thickness of the electric double layer. We therefore prepared larger crystals (up to $2 \mathrm{~mm}$.) - which could be ground afterwards to any desired size- by recrystallizing barium sulphate from hot concentrated sulphuric acid and subsequent ignition. The following results are typical :

Recrystallized barium sulphate, sample 1
Recrystallized barium sulphate, sample 3
Recrystallized barium sulphate, sample 4
Natural barite, sample $B$, ignited
Natural barite, sample $C$, Ignited

$$
\begin{gathered}
\zeta \text { in } \mathrm{mV} . \\
+24 \cdot 2 \\
+25 \cdot 0 ; 24 \cdot 3 \\
+26 \cdot 6 ; 27 \cdot 1 \\
+\quad 5 \cdot 4 ; 5 \cdot 3 \\
\quad+8 \cdot 4
\end{gathered}
$$

In spite of the fact that during preparation of recrystallized barium sulphate there was always an excess of sulphate ion, the potential is positive and well reproducible within $1 \mathrm{mV}$. for each sample. The ignition temperature $\left(500-1,000^{\circ} \mathrm{C}\right.$.) has no influence on the potential. Most electrolytes change the potential reversibly at room temperature; barium salts increase it, sulphates decrease it; but when, after treatment with the electrolyte solution, the potential is then measured against a saturated barium sulphate solution, the original value is always obtained. This value must therefore be regarded as a property of the sample. This behaviour is shown both by recrystallized barium sulphate and natural barite.

There is, however, a striking difference between the high potential of recrystallized barium sulphate and the much lower potential of natural barite. Spectroscopic analysis of our samples revealed that natural barite contains strontium and traces of calcium as impurities. However, these impurities cannot be responsible for the difference in potential. We in corporated such impurities into the recrystallized barium sulphate (during recrystallization); but, as a result, the potential was slightly increased, never decreased. Moreover, when natural barite is recrystallized from sulphuric acid, a high potential $(27 \mathrm{mV}$.) is obtained.

These and similar observations, which will be discussed in more detail elsewhere, lead us to the conclusion that the difference in potential between recrystallized barium sulphate and natural barite is not due to a difference in composition. The most likely cause is a difference in structure (geometrical or otherwise) of the surface. Natural barite, even after crushing, has even crystal surfaces, whereas recrystallized barium sulphate has very irregular surfaces. This is shown by an investigation in an optical as well as in an electron microscope, for the latter of which we are indebted to Dr. A. L. G. Rees and Mr. A. J. Hodge, of the Division of Industrial Chemistry of the Council for Scientific and Industrial Research. It is likely that the field of force is less well balanced in an irregular surface than in an even one.

Bikerman ${ }^{1}$ suggested that the $\zeta$-potential of a rough surface should be different from that of a smooth one for purely geometrical reasons. However, the distortion of the field of force in the surface as a factor influencing the electric potential at a surface is a somewhat unfamiliar concept in the field of electrokinetics. Nevertheless, besides our own observations, there are several findings of other investigators ${ }^{2,3}$ which suggest that the $\zeta$-potential of a crystalline solid is not solely determined by the composition of the system.

\section{A. S. BuchanaN \\ E. Heymann}

Chemistry Department,

University of Melbourne.

${ }^{2}$ Bikerman, J. Chem. Phys., 9, 88 (1941).

2 Kruyt and Ruyssen, Proc. Akad. Wetensch. Amsterdam, 37, 498 (1934).

' Wood, J. Amer. Chem. Soc., 68, 437 (1946). 